\title{
Glyceryl Monooleyl Ether-Based Liquid Crystalline Nanoparticles as a Transdermal Delivery System of Flurbiprofen: Characterization and in Vitro Transport
}

\author{
Tomonobu Uchino, ${ }^{*, a, b}$ Akiko Murata, ${ }^{a}$ Yasunori Miyazaki, ${ }^{a, b}$ Toshihiko Oka, ${ }^{c, d}$ and \\ Yoshiyuki Kagawa ${ }^{a, b}$ \\ ${ }^{a}$ Department of Clinical Pharmaceutics, School of Pharmaceutical Sciences, University of Shizuoka; 52-1 Yada \\ Suruga-ku, Shizuoka 422-8526, Japan: ${ }^{b}$ Laboratory of Clinical Pharmacokinetics, Shizuoka General Hospital; \\ 4-27-1 Kita-ando, Aoi-ku, Shizuoka 420-8527, Japan: ${ }^{c}$ Department of Physics, Graduate School of Science, Shizuoka \\ University; 836 Oya, Suruga-ku, Shizuoka 422-8529, Japan: and ${ }^{d}$ Nanomaterials Research Division, Research \\ Institute of Electronics, Shizuoka University; 836 Oya, Suruga-ku, Shizuoka 422-8529, Japan. \\ Received January 12, 2015; accepted February 17, 2015
}

Liquid crystalline nanoparticles (LCNs) were prepared using glyceryl monooleyl ether (GME) by the modified film rehydration method. Hydrogenated lecithin (HL), 1,3-butylene glycol (1,3-BG), and Poloxamer 407 were used as additives. The prepared LCN formulations were evaluated based on particle size, smallangle X-ray diffraction (SAXS) analysis, ${ }^{1} \mathrm{H}$ - and ${ }^{19} \mathrm{~F}$-NMR spectra, and in vitro skin permeation across Yucatan micropig skin. The composition (weight percent) of the LCN formulations were GME-HL-1,3-BG (4:1:15), 4\% GME-based LCN and GME-HL-1,3-BG (8:1:15), 8\% GME-based LCN and their mean particle sizes were $130-175 \mathrm{~nm}$. Flurbiprofen 5 and $10 \mathrm{mg}$ was loaded into 4\% GME-based LCN and 8\% GMEbased LCN systems, respectively. The results of SAXS and NMR suggested that both flurbiprofen-loaded formulations consist of particles with reverse type hexagonal phase (formation of hexosome) and flurbiprofen molecules were localized in the lipid domain through interaction of flurbiprofen with the lipid components. Flurbiprofen transport from the LCN systems across the Yucatan micropig skin was increased compared to flurbiprofen in citric buffer ( $\mathrm{pH}=3.0)$. The 8\% GME-based LCN systems was superior to the 4\% GME-based LCN for flurbiprofen transport. Since the internal hexagonal phase in the 8\% GME-based LCN systems had a higher degree of order compared to the 4\% GME-based LCN in SAXS patterns, the 8\% GME-based LCN system had a larger surface area, which might influence flurbiprofen permeation. These results indicated that the GME-based LCN system is effective in improving the skin permeation of flurbiprofen across the skin. NMR

Key words lyotropic liquid crystal; liquid crystalline nanoparticle; hexosome; skin permeation; flurbiprofen;

Under some circumstances, transdermal delivery may be superior to other routes of delivery due to advantages such as avoidance of first pass elimination and a lack of invasiveness. However, relatively few transdermal drugs have been commercialized owing to the efficient barrier function of the stratum corneum, which is located on the outer layer of the skin. To overcome the barrier function of the stratum corneum, several physical and chemical methods have been developed. ${ }^{1)}$

Recently, lyotropic liquid crystal (LLC) systems have been developed as a chemical method to overcome the stratum corneum barrier. ${ }^{2,3)}$ LLCs are usually composed of amphiphilic molecules and solvents that can form a variety of structures, including lamellar $\left(\mathrm{L}_{\alpha}\right)$, hexagonal (normal, or reverse) and inverted cubic (bicontinuous and micellar) structures. ${ }^{4)}$ Among these LLC systems, some of liquid crystal structures form a liquid crystalline nanoparticles (LCNs) by dispersing in an aqueous environment and the LCNs of reverse hexagonal phase and bicontinuous cubic phase are called "hexosomes $\left(\mathrm{H}_{\mathrm{II}}\right)$ " and "cubosomes," respectively.,

Glycerol monoolate (GMO) has been widely studied as an additive to form LLCs and their LCNs. ${ }^{6,7)}$ Effect of the additives such as block copolymer formation and electrostatic interaction were also investigated. ${ }^{8-11)}$ Cohen-Avrahami et al. reported transdermal delivery of sodium diclofenac with hexosomes of GMO in combination with cell-penetrating pep- tides. ${ }^{3,12)}$ Lopes et al. showed improved skin permeation for vitamin $\mathrm{K}$ and cyclosporine A with hexosomes of GMO. ${ }^{13,14)}$

However, it has been reported that the GMO liquid crystalline phase and its dispersions are sensitive to hydrolysis of the ester bond ${ }^{15)}$ and the action of hydrolytic enzymes like lipase. $^{16,17)}$

1-Glyceryl monooleyl ether (GME) is an ether analogue of GMO and has improved stability due to a lack of the ester bond found in GMO. ${ }^{18)}$ The chemical structure is shown in Fig. 1. ${ }^{19,20)}$

It was reported that GME formed a reverse hexagonal phase $^{18)}$ and a cubic phase in a water-propylene glycol mixture. $^{21)}$ However, few results have been reported on the use of GME-based LLC systems for transdermal delivery. ${ }^{20}$

The aim of this study was to prepare and characterize a

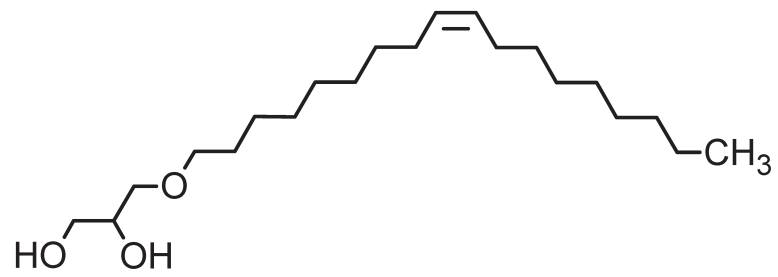

Fig. 1. Chemical Structure of 1-Glyceryl Monooleyl Ether 
GME-based liquid crystal for the transdermal delivery. Flurbiprofen, a non-steroidal anti-inflammatory drug used for the treatment of osteoarthritis and rheumatoid arthritis and which is also often used as a topical formulation, was used as a model drug. ${ }^{22}$ In current study, we prepared the LCNs of a flurbiprofen-loaded GME-based LCNs and characterized it using dynamic light scattering (DLS), small-angle X-ray diffraction (SAXS), and ${ }^{1} \mathrm{H}$ - and ${ }^{19} \mathrm{~F}-\mathrm{NMR}$. Flurbiprofen transport across the Yucatan micropig skin was also investigated.

\section{Experimental}

Materials Flurbiprofen was purchased from Cayman Chemical (Ann Arbor, MI, U.S.A.). 1-Glyceryl monooleyl ether (GME) and hydrogenated lecithin (HL) were kindly donated by Nikko Chemicals Co., Ltd. (Tokyo, Japan). Poloxamer 407 and 1,3-butylene glycol (1,3-BG) were obtained from BASF Japan Ltd. (Tokyo, Japan) and Wako Pure Chemical Industries, Ltd. (Tokyo, Japan), respectively. All other chemicals used were of reagent grade.

Preparation of LCNs GME and flurbiprofen were dissolved in ethanol. HL was dissolved in chloroform. These solutions were then mixed in the sample tube at the appropriate molar ratio. The organic solvents were removed by rotary evaporation (CVE-200D, Tokyo Rikakikai Co., Ltd., Tokyo, Japan) under vacuum at $30^{\circ} \mathrm{C}$. The dry lipid film was hydrated with $1 \mathrm{~mL}$ of $50 \mathrm{~mm}$ citrate buffer ( $\mathrm{pH} \mathrm{3.0)}$ containing the appropriate amounts of 1,3-BG and Poloxamer 407 (1\%). For the LCNs prepared for NMR measurements, the film was hydrated with $50 \mathrm{~mm}$ citrate buffer $(\mathrm{pH} 3.0)$ using $\mathrm{D}_{2} \mathrm{O}$, which contained 1,3-BG and Poloxamer 407. The suspensions were subsequently sonicated for 1 min using a Branson Sonifier 250 (Branson Ultrasonics, Emerson Japan Ltd., Kanagawa, Japan). During preparation, sample tubes were cooled with ice.

Determination of the Saturated Concentration of Flurbiprofen in the LCNs To determine the saturated concentration of flurbiprofen, several LCN formulations were prepared with increasing flurbiprofen concentrations. The formulations were stored in the dark. Samples were viewed with polarization microscopy $14 \mathrm{~d}$ after preparation to determine if flurbiprofen crystals had formed. The saturated concentration of flurbiprofen was selected as the formulation that contained the highest flurbiprofen concentration without flurbiprofen crystal formation after the 14-d storage period. ${ }^{23)}$

SAXS Measurement SAXS measurements were performed on a laboratory system (NANO-Viewer system, Rigaku, Japan). The rotating copper anode generator was operated at $40 \mathrm{kV}$ and $30 \mathrm{~mA}$. The two-dimensional scattering pattern was measured using a two-dimensional detector (PILATUS 100K, Dectris, Baden, Switzerland). The sampledetector distance was set to about $472 \mathrm{~mm}$, which was calibrated with silver behenate. The scattering intensity, $I(S)$, was measured for scattering angles $(S=2 \sin \theta / \lambda)$ ranging from 0.03 to $0.56 \mathrm{~nm}^{-1}$. The sample was added to a polyimide tube of $1.0 \mathrm{~mm}$ in diameter. The temperature was maintained at $32^{\circ} \mathrm{C}$ for each measurement.

Particle Size Measurement Particle size and polydispersity index (PDI) of all LCN formulations were measured by dynamic light scattering (DLS) using Zetasizer Nano ZX (Malvern Instruments, Malvern, U.K.). The analysis was performed at a scattering angle of $90^{\circ}$ and a temperature of $25^{\circ} \mathrm{C}$. Before measurement, all samples were diluted with $50 \mathrm{~mm}$ citrate buffer ( $\mathrm{pH}$ 3.0). The PDI describes the homogeneity of the particle size in the samples. A stability study was performed to monitor particle size during a period of $14 \mathrm{~d}$ after preparation.

${ }^{\mathbf{1}} \mathbf{H}$ - and ${ }^{19} \mathbf{F}-\mathbf{N M R}$ Studies ${ }^{1} \mathrm{H}$ - and ${ }^{19} \mathrm{~F}-\mathrm{NMR}$ experiments with flurbiprofen-loaded LCN systems were performed at $500 \mathrm{MHz}$ on a JEOL ECA-500 NMR spectrometer (JEOL, Tokyo, Japan). As a reference, flurbiprofen was dissolved in phosphate-buffered saline (PBS) prepared with $\mathrm{D}_{2} \mathrm{O}(1.2 \mathrm{mg} /$ $\mathrm{mL}$ ). All measurements were performed at $32^{\circ} \mathrm{C}$ (typical skin surface temperature). For the ${ }^{19} \mathrm{~F}-\mathrm{NMR}$ study, data were recorded with 500 scans using a relaxation delay of $5 \mathrm{~s}$. A total of 8192 data points were sampled, leading to an acquisition time of $0.46 \mathrm{~s}$. A sealed glass capillary with a small amount of $10 \mu \mathrm{g} / \mathrm{mL}$ trifluoroacetic acid in $\mathrm{D}_{2} \mathrm{O}$ solution was added to the NMR sample tube and the $\mathrm{CF}_{3}$ signal $(-76.5 \mathrm{ppm})$ was measured as an internal standard.

In Vitro Skin Permeation Studies In vitro transport studies were performed using a flow diffusion cell array system (Introtec, Kanagawa, Japan). Excised Yucatan micropig skin (YMP) skin was used as model skin. Full-thickness YMP skin, previously stored in a freezer at $-80^{\circ} \mathrm{C}$, was slowly thawed and any subcutaneous fat was removed using surgical scissors. The skin was sliced into approximately $600-\mu \mathrm{m}$ thick slices by using a Linear Slicer ${ }^{\circledR}$ PRO7 (Dosaka EM Co., Ltd., Kyoto, Japan), and hydrated in cold PBS ( $\mathrm{pH}$ 7.4) prior to the experiment. The donor compartment of the diffusion cell was filled with $50 \mu \mathrm{L}$ of the formulation. The formulation was applied occlusively. The diffusion surface area was $1.1 \mathrm{~cm}^{2}$. A saturated buffer solution of flurbiprofen (citric buffer, $\mathrm{pH} 3.0$ ) served as a control. The acceptor compartment was filled with PBS (pH 7.4), which was perfused at a flow rate of $1.0 \mathrm{~mL} / \mathrm{h}$. The acceptor compartment was kept at $37^{\circ} \mathrm{C}$, maintaining the skin surface temperature at around $32^{\circ} \mathrm{C}$. Samples were collected every hour over a 24 -h period. Flurbiprofen concentrations in the donor and acceptor phases were measured by HPLC as described below.

HPLC Analysis The HPLC system consisted of a pump (LC-10AT, Shimadzu, Kyoto, Japan), an auto-injector (SIL10AXL, Shimadzu), a UV detector (SPD-10A, Shimadzu), and an analyzer (CLASS LC-10, Shimadzu). The column was a Synergi $4 \mathrm{u}$ Fusion-RP $80 \mathrm{~A}(150 \times 4.60 \mathrm{~mm}$, Phenomenex, Torrance, CA, U.S.A.). The mobile phase was composed of methanol/50 mM phosphate buffer $(\mathrm{pH} 2.8)$ at a ratio of $75: 25$ $(\mathrm{v} / \mathrm{v})$. The flow rate was set at $0.8 \mathrm{~mL} / \mathrm{min}$. The UV detection wavelength was set at $254 \mathrm{~nm}$. This system yielded a retention time of $6.3 \mathrm{~min}$ for flurbiprofen. The lower limit of detection for flurbiprofen was $800 \mathrm{pg}$. A standard curve of flurbiprofen was prepared to allow the calculation of flurbiprofen in experimental samples. Samples of the receptor compartment solution $(100 \mu \mathrm{L})$ were injected into the HPLC for determination of flurbiprofen flux across YMP skin.

Statistical Analysis Permeation data obtained after $24 \mathrm{~h}$ (expressed as the mean \pm S.E.M.) were analyzed by Tukey's multiple comparison tests and ANOVA. Statistical analysis was carried out using SPSS software version 17.0 (SPSS Inc., Chicago, IL, U.S.A.). A $p$ value of $<0.05$ was considered to be significant. 


\section{Results and Discussion}

Characterization and Physicochemical Properties of Flurbiprofen Loaded GME-Based LCN Systems In this study, a buffer with $\mathrm{pH} 3.0$ was selected as a dispersing solution for the LCN preparation since the focus was primarily on flurbiprofen in the LCN systems. The $\mathrm{p} K_{\mathrm{a}}$ of flurbiprofen has been reported to be $4.2 .^{24)}$ Therefore, at this $\mathrm{pH}$ (3.0), most of the flurbiprofen is in its unionized form and is expected to be localized in the lipid domain in the GME-based liquid crystal.

Table 1 summarizes the formulation and particle characterization of GME-based liquid crystal dispersions.

Mean particle size ( $Z$-average) ranged from 130 to $175 \mathrm{~nm}$. The PDI in all formulations was between 0.20 and 0.29 , indicating that all systems are homogeneous $(0.0$ is very homogeneous, 1.0 is very heterogeneous). Flurbiprofen loading did not affect particle size. No change in $Z$-average size or PDI was observed after the preparations were stored for two weeks. Flurbiprofen 5 and $10 \mathrm{mg}$ was loaded in the 4\% GME-based LCNs and 8\% GME-based LCNs, respectively. From the results of polarization microscopy, the saturated concentrations of flurbiprofen were higher in the LCN formulations than the saturated concentration of flurbiprofen $(70 \mu \mathrm{g} / \mathrm{mL})$ in citrate buffer $(\mathrm{pH} 3.0){ }^{23)}$ These results suggested that flurbiprofen molecules were entrapped in LCNs and interacted with LLC components.

To characterize the structure of GME-based nanoparticles, SAXS measurements were performed on the prepared LCNs. The SAXS patterns and lattice parameters are shown in Fig. 2 and Table 2, respectively.

In the sample with empty the 4\% GME-based LCN system, a broad diffraction pattern was observed (Fig. 2(a)). On the other hand, weak diffraction peaks at $S=0.184,0.319$, and $0.366 \mathrm{~nm}^{-1}$ were observed in the flurbiprofen-loaded $4 \%$ GME-based LCN system (flurbiprofen content: $5 \mathrm{mg}$ ). Unassigned broad peaks are observed in at $0.14 \mathrm{~nm}^{-1}$ (indicated by an asterisk) in Figs. 2(a)-(c). In the sample with empty the $8 \%$ GME-based LCN system, weak diffraction peaks at 0.182 , 0.317 , and $0.367 \mathrm{~nm}^{-1}$ were observed.

Sharp diffraction peaks were observed in the flurbiprofenloaded 8\% GME-based LCN system (flurbiprofen content: $10 \mathrm{mg}$ ) at $0.194,0.335$, and $0.387 \mathrm{~nm}^{-1}$. The space group ratio in Figs. 2(b)-(d) was found to be $1: \sqrt{3}: 2$. These results in-

Table 1. Characterization of Glyceryl Monooleyl Ether (GME)-Based Liquid Crystalline Nanoparticle Systems

\begin{tabular}{ccccc}
\hline \hline Formulation & Composition (wt\%) & Flurbiprophen concentration $(\mathrm{mg} / \mathrm{mL})$ & Z-Average (d.nm \pm S.D.) & PDI ( \pm S.D.) \\
\hline GME-HL-1.3-BG & $4: 1: 15$ & - & $134.7 \pm 47.0$ & $0.28 \pm 0.20$ \\
& $4: 1: 15$ & 5.0 & $149.2 \pm 12.4$ & $0.28 \pm 0.05$ \\
& $8: 1: 15$ & - & $165.2 \pm 5.5$ & $0.24 \pm 0.05$ \\
& $8: 1: 15$ & 10.0 & $171.7 \pm 20.4$ & $0.22 \pm 0.07$ \\
\hline
\end{tabular}

Values are the means \pm S.D. $(n=3) . \mathrm{HL}=$ hydrogenated lecithin; $1,3-\mathrm{BG}=1,3$-butylene glycol; $Z$-Average=intensity weighted harmonic mean particle size; $\mathrm{PDI}=$ polydispersity index, a measure of the homogeneity of particle sizes. Values range from 0.0 (very homogenous) to 1.0 (very heterogeneous).

(a)

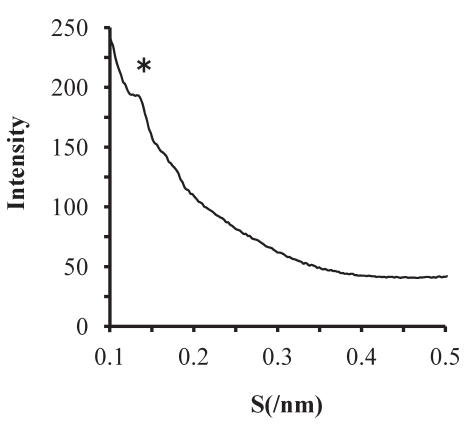

(b)

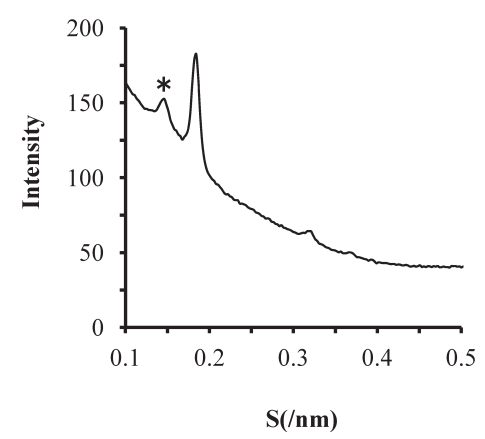

(c)

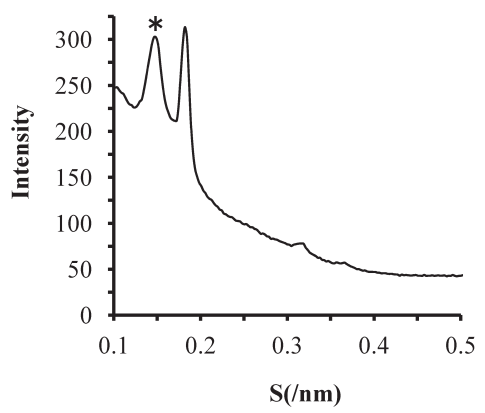

(d)

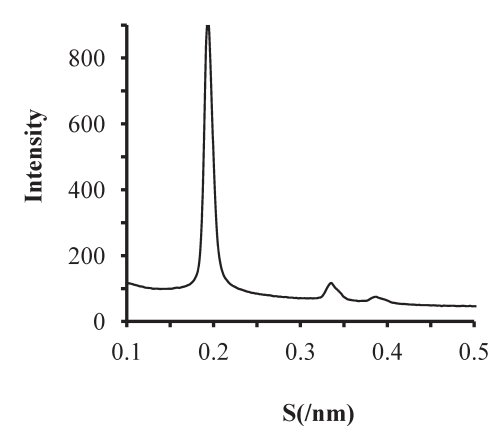

Fig. 2. Small Angle X-Ray Diffraction (SAXS) Patterns of Glyceryl Monooleyl Ether (GME)-Based Liquid Crystalline Nanoparticle (LCN) System

Empty 4\% GME-based LCNs (a), flurbiprofen-loaded 4\% GME-based LCNs (b), empty 8\% GME-based LCNs (c), flurbiprofen-loaded 8\% GME-based LCNs (d). Each weak broad peak at $0.14 \mathrm{~nm}^{-1}$ (as indicated by asterisk) in Figs. 2(a)-(c) represent as unassigned peak except for hexagonal structure. 
Table 2. Lattice Parameters of Glyceryl Monooleyl Ether (GME)-Based Liquid Crystalline Nanoparticle Systems

\begin{tabular}{|c|c|c|c|c|c|c|}
\hline Formulation & Composition (wt\%) & Flurbiprophen concentration $(\mathrm{mg} / \mathrm{mL})$ & hk & Peak position $(\mathrm{s} / \mathrm{nm})$ & Spacing ratio & Interlayer spacing $(\mathrm{nm})$ \\
\hline \multirow[t]{12}{*}{ GME-HL-1.3-BG } & $4: 1: 15$ & - & - & - & - & - \\
\hline & & & - & - & - & - \\
\hline & & & - & - & - & - \\
\hline & $4: 1: 15$ & 5.0 & 10 & 0.184 & 1.00 & 6.26 \\
\hline & & & 11 & 0.319 & 1.73 & 6.27 \\
\hline & & & 20 & 0.366 & 1.99 & 6.31 \\
\hline & $8: 1: 15$ & - & 10 & 0.182 & 1.00 & 6.34 \\
\hline & & & 11 & 0.317 & 1.74 & 6.32 \\
\hline & & & 20 & 0.367 & 2.01 & 6.31 \\
\hline & $8: 1: 15$ & 10 & 10 & 0.194 & 1.00 & 5.96 \\
\hline & & & 11 & 0.335 & 1.73 & 5.96 \\
\hline & & & 20 & 0.387 & 2.00 & 5.96 \\
\hline
\end{tabular}

$\mathrm{hk}=$ Miller indices; $\mathrm{HL}=$ hydrogenated lecithin; 1,3-BG=1,3-butylene glycol.

(a)

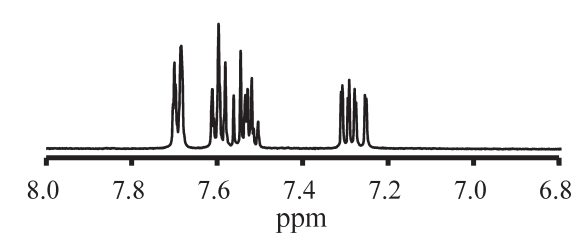

(b)

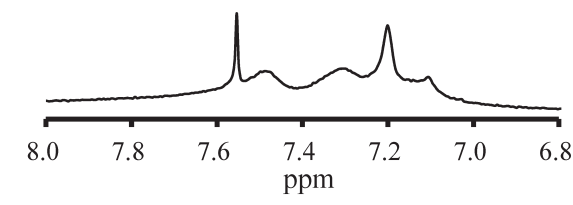

(c)

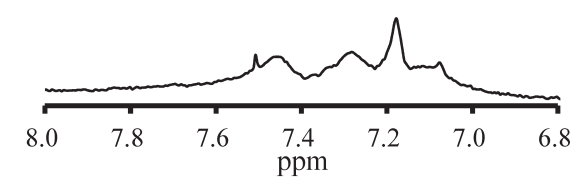

(d)

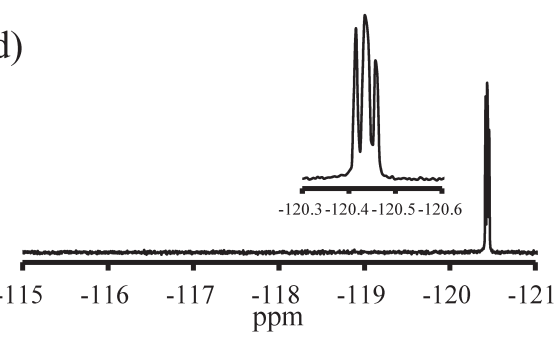

(e)

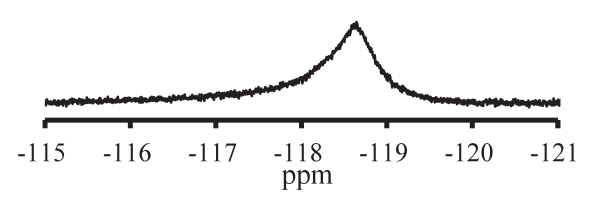

(f)

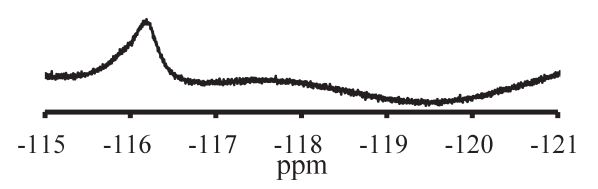

Fig. 3. ${ }^{1} \mathrm{H}-$ (Left Side) and ${ }^{19}$ F-NMR (Right Side) Spectra of Glyceryl Monooleyl Ether (GME)-Based Liquid Crystalline Nanoparticle (LCN) Systems

Flurbiprofen in phosphate buffered saline solution (a) and (d), flurbiprofen-loaded 4\% GME-based LCNs (b) and (e), flurbiprofen-loaded 8\% GME-based LCNs (c) and (f).

dicated that these both flurbiprofen loaded LCNs formed a reverse hexagonal structure and both formulations are hexosome. ${ }^{21,25)}$

It was reported that the self-assembled nanostructure of similar lipids inducing non-lamellar phases such as GMO is independent of water content under full hydration conditions. ${ }^{26)}$ Therefore, the weak signal in Fig. 2(a) might be attributed to the need of a longer exposure of the sample. And the coexistence of the hexagonal structure with another phase might be formed in the lower degree of hexagonal structure in unassigned broad peaks at $0.14 \mathrm{~nm}^{-1}$ (indicated by an asterisk) in Figs. 2(a)-(c).

In the $4 \%$ GME-based LCN system, flurbiprofen loading induced the change from a no repeat structure to a hexagonal structure. Increased intensity of the diffraction peaks due to the hexagonal structure and its lattice parameter changed from 6.3 to $6.0 \AA$, in $8 \%$ GME-based LCN system. It was reported that encapsulated molecules such as bupivacaine, lidocaine, and propranole affected the liquid crystalline structure, and under some conditions, these molecules induced a phase transition. ${ }^{27-29)}$ Similarly, results from the current study indicated that the flurbiprofen molecule participated in the stabilizing the hexagonal structure in GME-based LCN system by the interacting with the lipid components and contributed to the formation of hexagonal structure.

It has been reported that reverse hexagonal structures are 
closed and have extended micellar columnar structures. ${ }^{4)}$ It is possible that a hydrophilic molecule can be entrapped in the internal water domain, whereas hydrophobic molecules will be located within the lipid domain. To investigate the molecular state of flurbiprofen in the GME-based hexosomes and interaction between flurbiprofen and lipid components of the hexosomes, NMR measurements were carried out. The results of ${ }^{1} \mathrm{H}-\mathrm{NMR}$ in the aromatic region and ${ }^{19} \mathrm{~F}-\mathrm{NMR}$ spectra were shown in Fig. 3.

In the flurbiprofen solution, sharp signals of the aromatic proton of flurbiprofen were observed due to the rapid molecular motion. However, the aromatic proton signals were significantly broader in the liquid crystalline samples and were shifted upfield (Figs. 3(b), (c)).

Previously studies showed that broadening of the signal broadening from aromatic protons in drug molecules was caused by entrapment in the bilayer of surfactant-based vesicles. $^{23,30,31)}$ Therefore, the results from the present study indicated that flurbiprofen molecules were trapped in the lipid domain of hexagonal liquid crystal by the interaction with lipid components and their mobility was restricted.

The same tendency was observed with the fluorine signal of flurbiprofen. Figures 3(d)-(f) show ${ }^{19}$ F-NMR spectra of flurbiprofen loaded liquid crystal systems. In the flurbiprofen solution, sharp signals due to the rapid molecular motion were observed from 120.3 to $120.6 \mathrm{ppm}$. Broader signals and peak shifts were observed in the both liquid crystalline samples. These results indicated that the biphenyl ring in flurbiprofen molecule was entrapped in the lipid domain of the hexagonal structure by the interaction with lipid components. Figure 4 shows ${ }^{1} \mathrm{H}-\mathrm{NMR}$ spectra of the olefin region in the GME-based LCN systems.

In the GME solution, a sharp signal was observed at $5.32 \mathrm{ppm}$ due to rapid molecular motion. In all liquid crystalline samples, olefin signals were found to broaden and flurbiprofen loading into the liquid crystalline samples resulted in an upfield shift. Cohen-Avrahami et al. reported that sodium diclofenac incorporated at the interface of GMO-based cubic and lamellar mesophase by interacting with GMO, which induced denser packing. ${ }^{32,33)}$ They speculated that diclofenac acts as "structure stabilizer" in this system, and that the characteristics of the system depend on the incorporated

(a)

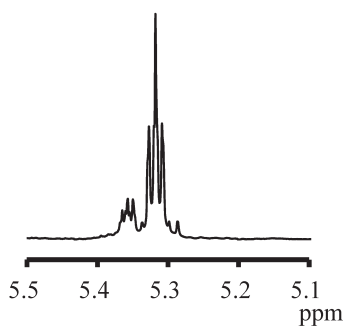

(b)

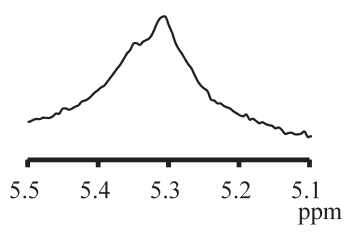

(d)

(c)

(e)
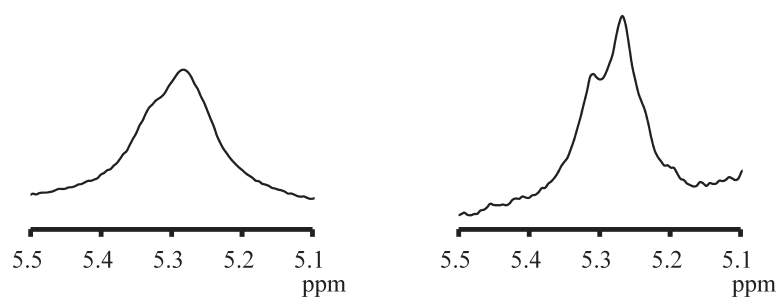

Fig. 4. ${ }^{1} \mathrm{H}-\mathrm{NMR}$ Spectra of Olefinic Region in the Glyceryl Monooleyl Ether (GME)-Based Liquid Crystalline Nanoparticle (LCN) Systems

GME $\mathrm{CD}_{3} \mathrm{OD}$ solution (a), empty 4\% GME-based LCNs (b), flurbiprofen-loaded $4 \%$ GME-based LCNs (c), empty $8 \%$ GME-based LCNs (d), flurbiprofen-loaded $8 \%$ GME-based LCNs (e).

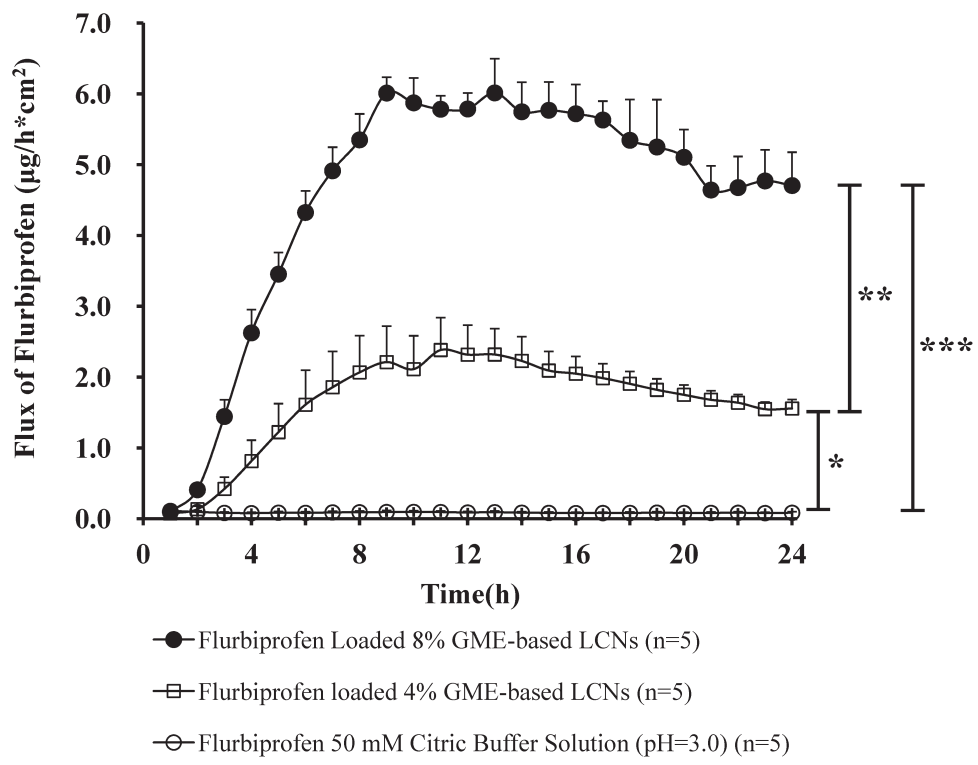

Fig. 5. The Flux Curves for Flurbiprofen from 4\% and 8\% Glyceryl Monooleyl Ether (GME)-Based Liquid Crystalline Nanoparticles (LCNs) and $50 \mathrm{~mm}$ Citric Buffer Solution across Yucatan Micropig Skin 
molecules. As previously mentioned, flurbiprofen in existed in the unionized form in this system $(\mathrm{pH} 3.0)$ and showed hydrophobic properties.

Therefore, the SAXS and NMR results indicated that flurbiprofen incorporated in the lipid domain of the hexagonal structure by interacting with the olefin portion of the GME molecules, which dramatically decreased the mobility of flurbiprofen molecules.

Skin Permeation of Flurbiprofen from GME-Based LCNs Figure 5 shows the flux curve of flurbiprofen from GME-based LCN systems and effect of less (4\% GME-based LCNs) and more ordered hexosomes (8\% GME-based LCNs) on flurbiprofen permeation.

These results showed that flurbiprofen permeation from hexosomes was facilitated by the entrapment of flurbiprofen in the lipid domain of the hexagonal structure. The highly ordered hexosome system ( $8 \%$ GME-based LCNs) showed higher flurbiprofen flux across YMP skin. The amount of flurbiprofen that permeated across the YMP skin from $50 \mathrm{~mm}$ citric buffer, flurbiprofen-loaded 4\% GME-based LCNs and flurbiprofen-loaded $8 \%$ GME-based LCNs were $0.08 \pm 0.01$, $1.56 \pm 0.13$, and $4.71 \pm 0.47 \mu \mathrm{g} / \mathrm{h} \cdot \mathrm{cm}^{2}$, respectively. From the result of Fig. 5, cumulative amount of flurbiprofen permeated (24h) from $50 \mathrm{~mm}$ citric buffer, $4 \%$ and $8 \%$ GME-based LCNs were calculated and their results were $1.74 \pm 0.3,51.3 \pm 4.9$, and $109 \pm 6.5 \mu \mathrm{g} / \mathrm{cm}^{2}$, respectively.

The detail mechanism of drug permeation across the skin by LCN system is not fully understood. In cubosome system, it was speculated that cubic structure with similar nano-structure as the skin, increase the interaction between skin and formulation and enhances the skin permeation. ${ }^{5,20)}$ Lopes et al. reported that hexagonal phase may facilitate the fusion of the LCNs with stratum corneum and deeper skin layers and thereby may improve drug delivery to the skin. ${ }^{14)}$ Moreover, they speculated that the larger surface area of the hexosome system might have resulted in enhanced contact with the porcine ear skin that improved vitamin $\mathrm{K}$ permeation. ${ }^{13)}$ Since both flurbiprofen-loaded GME/HL/1,3-BG systems also formed hexosomes, it was concluded that nanoparticles of larger surface area were formed in both formulations. Flurbiprofen transport in hexosomes with higher GME content $(8 \%)$ was higher than transport in the system with lower GME content (4\%). Both GME-based LCN systems were considered to be saturated with flurbiprofen, resulting in maximum thermodynamic activities. Since the hexagonal structure in the $8 \%$ GME-based LCN system had a higher degree of order compared to the $4 \%$ system in SAXS patterns, the particles in the $8 \%$ GME-based LCN system had a larger surface area, which may influence flurbiprofen permeation through YMP skin. Further investigations are required to understand the permeation mechanism using GME-based LCN systems.

\section{Conclusion}

Flurbiprofen transport across the YMP skin was increased by loading flurbiprofen into GME-based LCNs. These studies suggested that flurbiprofen molecules interacted with LLC components and their mobility was restricted in the hydrophobic domain of the hexagonal structure. This study is the first characterization of GME-based LCN systems and includes initial studies on the flux of a model drug, flurbiprofen, across YMP skin. Detailed experiments on the mechanisms of skin transport may be addressed in future studies.

Acknowledgments This study was partially supported by Grant-in-Aid for Scientific Research (C) (JSPS, 26460225) from the Japan Society for the Promotion of Sciences. SAXS measurements were carried out at the Center for Instrumental Analysis of Shizuoka University.

Conflict of Interest The authors declare no conflict of interest.

\section{References}

1) Williams A., "Transdermal and Topical Drug Delivery: From Theory to Clinical Practice," Pharmaceutical Press, London, 2003.

2) Yamada K., Yamashita J., Todo H., Miyamoto K., Hashimoto S., Tokudome Y., Hashimoto F., Sugibayashi K., J. Oleo Sci., 60, 31-40 (2011).

3) Cohen-Avrahami M., Aserin A., Garti N., Colloids Surf. B Biointerfaces, 77, 131-138 (2010).

4) Guo C., Wang J., Cao F., Lee R. J., Zhai G., Drug Discov. Today, 15, 1032-1040 (2010)

5) Esposito E., Cortesi R., Drechsler M., Paccamiccio L., Mariani P., Contado C., Stellin E., Menegatti E., Bonina F., Puglia C., Pharm. Res., 22, 2163-2173 (2005).

6) Larsson K., Curr. Opin. Colloid In., 5, 64-69 (2000).

7) Caboi F., Amico G. S., Pitzalis P., Monduzzi M., Nylander T., Larsson K., Chem. Phys. Lipids, 109, 47-62 (2001).

8) Amar-Yuli I., Wachtel E., Shoshan E. B., Danino D., Aserin A., Garti N., Langmuir, 23, 3637-3645 (2007).

9) Yaghmur A., Sartori B., Rappolt M., Phys. Chem. Chem. Phys., 13, 3115-3125 (2011).

10) Yariv D., Efrat R., Libster D., Aserin A., Garti N., Colloids Surf. B Biointerfaces, 78, 185-192 (2010).

11) Yaghmur A., Larsen S. W., Schmitt M., Ostergaard J., Larsen C., Jensen H., Urtti A., Rappolt M., Soft Matter, 7, 8291-8295 (2011).

12) Cohen-Avrahami M., Libster D., Aserin A., Garti N., J. Phys. Chem. B, 115, 10189-10197 (2011).

13) Lopes L. B., Speretta F. F., Bentley M. V., Eur. J. Pharm. Sci., 32, 209-215 (2007).

14) Lopes L. B., Ferreira D. A., de Paula D., Garcia M. T., Thomazini J. A., Fantini M. C., Bentley M. V., Pharm. Res., 23, 1332-1342 (2006).

15) Monduzzi M., Ljusberg-Wahren H., Larsson K. R., Langmuir, 16, 7355-7358 (2000).

16) Borné J., Nylander T., Khan A., Langmuir, 18, 8972-8981 (2002).

17) Borné J., Nylander T., Khan A., J. Phys. Chem. B, 106, 1049210500 (2002)

18) Barauskas J., Svedaite I., Butkus E., Razumas V., Larsson K., Tiberg F., Colloids Surf. B Biointerfaces, 41, 49-53 (2005).

19) Nguyen T.-H., Hanley T., Porter C. J. H., Boyd B. J., Drug Deliv. Transl. Res., 1, 429-438 (2011).

20) Evenbratt H., Jonsson C., Faergemann J., Engstrom S., Ericson M. B., Int. J. Pharm., 452, 270-275 (2013).

21) Engström S., Wadsten-Hindrichsen P., Hernius B., Langmuir, 23, 10020-10025 (2007).

22) Fang J. Y., Hwang T. L., Leu Y. L., Int. J. Pharm., 250, 313-325 (2003).

23) Uchino T., Matsumoto Y., Murata A., Oka T., Miyazaki Y., Kagawa Y., Int. J. Pharm., 464, 75-84 (2014).

24) Anderson B. D., Conradi R. A., J. Pharm. Sci., 74, 815-820 (1985).

25) Yaghmur A., Glatter O., Adv. Colloid Interface, 147-148, 333-342 (2009).

26) de Campo L., Yaghmur A., Sagalowicz L., Leser M. E., Watzke H., Glatter O., Langmuir, 20, 5254-5261 (2004).

27) Engström S., Engström L., Int. J. Pharm., 79, 113-122 (1992). 
28) Chang C. M., Bodmeier R., Int. J. Pharm., 147, 135-142 (1997).

29) Yaghmur A., Rappolt M., Ostergaard J., Larsen C., Larsen S. W. Langmuir, 28, 2881-2889 (2012).

30) Uchino T., Lefeber F., Gooris G., Bouwstra J., Int. J. Pharm., 412, 142-147 (2011).

31) Uchino T., Lefeber F., Gooris G., Bouwstra J., Eur. J. Pharm. Bio- pharm., 86, 156-166 (2014).

32) Cohen-Avrahami M., Shames A. I., Ottaviani M. F., Aserin A., Garti N., Colloids Surf. B Biointerfaces, 122, 231-240 (2014).

33) Cohen-Avrahami M., Shames A. I., Ottaviani M. F., Aserin A., Garti N., J. Phys. Chem. B, 118, 6277-6287 (2014). 